\title{
An Entropy-Based Morphological Analysis of River Basin Networks
}

\author{
Mauro Fiorentino and Pierluigi Claps \\ Dipartimento di Ingegneria e Fisica dell'Ambiente, Università della Basilicata, Via della Tecnica, Potenza, Italy \\ VIJAY P. SINGH
}

Department of Civil Engineering, Louisiana State University, Baton Rouge

\begin{abstract}
Under the assumption that the only information available on a drainage basin is its mean elevation, the connection between entropy and potential energy is explored to analyze drainage basins morphological characteristics. The mean basin elevation is found to be linearly related to the entropy of the drainage basin. This relation leads to a linear relation between the mean elevation of a subnetwork and the logarithm of its topological diameter. Furthermore, the relation between the fall in elevation from the source to the outlet of the main channel and the entropy of its drainage basin is found to be linear and so is also the case between the elevation of a node and the logarithm of its distance from the source. When a drainage basin is ordered according to the Horton-Strahler ordering scheme, a linear relation is found between the drainage basin entropy and the basin order. This relation can be characterized as a measure of the basin network complexity. The basin entropy is found to be linearly related to the logarithm of the magnitude of the basin network. This relation leads to a nonlinear relation between the network diameter and magnitude, where the exponent is found to be related to the fractal dimension of the drainage network. Also, the exponent of the power law relating the channel slope to the network magnitude is found to be related to the fractal dimension of the network. These relationships are verified on three drainage basins in southern Italy, and the results are found to be promising.
\end{abstract}

\section{INTRODUCTION}

A multitude of definitions of entropy, corresponding to different uses, have been reported in the scientific literature [Wilson, 1970]. All of these definitions can, however, be traced to one of the three types of entropies: (1) thermodynamic entropy [e.g., Prigogine, 1967] that forms the basis of the second law of thermodynamics, (2) statistical mechanical entropy [Boltzmann, 1872], and (3) informational entropy [Shannon, 1948]. The thermodynamic entropy and statistical mechanical entropy are related and can simply be clubbed as physical entropy. One can also designate physical entropy as Boltzmann entropy and informational entropy as Shannon entropy. The link between physical entropy and informational entropy has thus far been elusive. A distinction can, however, be made between physical entropy as an objective measure of some property of a system, and informational entropy as a subjective concept for use as a model-building tool to maximize the use of available information [Wilson, 1970]. This distinction is important in that developments in hydrology and water resources employing the entropy concepts have occurred along the separated lines of physical and informational entropies, without exploiting the relationship between them.

Nearly 30 years ago, Leopold and Langbein [1962] applied for the first time the concepts of physical entropy to study the behavior of streams. Their application was based on the analogy between heat energy and temperature in a thermodynamic system and potential energy and elevation, respectively, in a stream system. Two thermodynamic principles were applied. The first principle is that the most probable state of a system is the one of maximum entropy. The second

Copyright 1993 by the American Geophysical Union.

Paper number 92WR02332.

0043-1397/93/92WR-02332\$05.00 is the principle of minimum entropy production rate. Using these principles, Yang [1971] derived for a stream system the law of average stream fall, and the law of the least rate of energy expenditure. Yang and coworkers (see, for example, Song and Yang [1990] for a recent review) and others have since applied the latter law to a range of problems in hydraulics. The connection between entropy and potential energy, which these workers so successfully exploited to investigate river engineering, sediment transport, and other problems, was not exploited in hydrology. In this study we pursue this connection to derive relations between entropy and mean elevation for a drainage basin network and to derive relations for the river profiles.

Much of the work employing the entropy concepts in hydrology has been with the application of informational entropy. The beginnings of such a work can be traced to Lienhard [1964], who used a statistical mechanical approach to derive a dimensionless unit hydrograph of a drainage basin. Implicit in Lienhard's derivation was the concept underlying the maximum entropy formalism developed by Jaynes [1957]. It was, however, Sonuga [1972], who was the first to explicitly use the principle of maximum entropy to derive a flood frequency distribution, subject to the information on moments of flood values. During this intervening period of nearly 20 years, the concepts of informational entropy have been applied to a wide range of problems in hydrology and water resources (see, e.g., Singh and Fiorentino [1992] for a recent review). These applications have, however, lacked the physical content unlike the parallel applications in hydraulics and have been mostly statistical in character.

Beginning with the pioneering work of Rodriguez-Iturbe and Valdes [1979] and Gupta et al. [1980], a considerable amount of work has been done on application of geomorphology to the understanding of drainage basin response. 


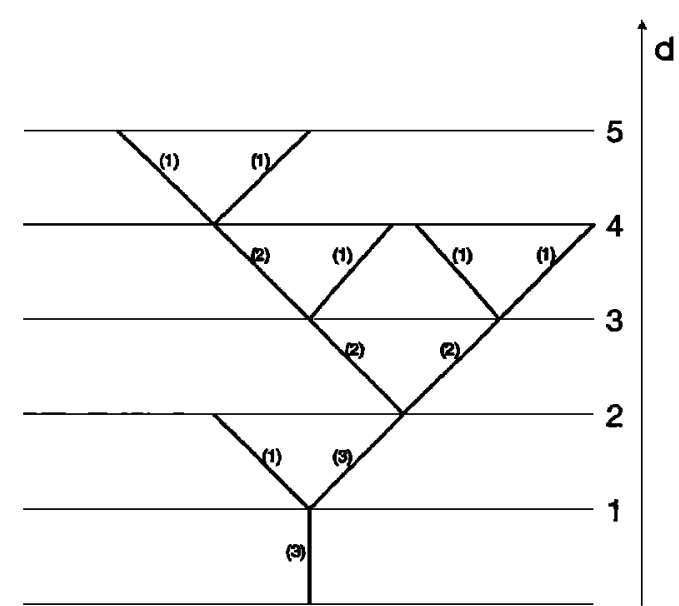

Fig. 1. Definition sketch of a simplified drainage network formed by links, external nodes (sources) and internal nodes. The Horton order $\omega$ of each link is reported in parenthesis. In this network the magnitude $n$ (number of sources) is 6 , the topological diameter $D$ (maximum topological distance $d$ from the outlet) is 5 , and the Horton order $\Omega$ (maximum value of $\omega$ ) is 3 .

The desire to understand hydrologic response at the basin scale has simultaneously led to significant advances in understanding river basin morphological characteristics (see the collection of papers edited by Gupta et al. [1986]). The entropy concepts were, however, not employed in these studies, despite Yang's [1971] work in precisely the same area.

Only recently, Rodriguez-Iturbe et al. [1992], by using the principles of minimum energy dissipation in a river reach and of uniform energy expenditure per unit area of channel, explained some widely observed empirical laws relating geometrical characteristics of the river network system. Furthermore, the recognition of the fractal structure of river networks [Tarboton et al., 1988; La Barbera and Rosso, 1989; Rosso et al., 1991; Marani et al., 1991] points out that river systems tend to evolve into a critical state with no characteristic time or length scale.

In this study we explore the relationship of entropy to certain morphological characteristics of drainage-basin networks. This paper is organized as follows. First, entropy of a drainage basin network is defined. Then, the connection between entropy and potential energy is explored to analyze river basin morphological characteristics. Specifically, in section 2, informational, primary, and secondary entropies are derived. A short discussion of drainage basin data that will be used to verify the relations to be derived in the ensuing sections is given in section 3 . In section 4 we derive a relation between mean elevation and entropy and a relation between mean elevation of the network and that of its subnetworks. In section 5 we investigate river profiles by using entropy. The relation between entropy and the Strahler order, basin magnitude and fractal dimension constitutes the subject matter of section 6 . Verification of the relations is undertaken using the data from three drainage basins in southern Italy in the individual sections of the paper.

\section{Informational, Primary, and Secondary Entropies}

A drainage network can be schematized by the channel network in that channels are idealized as single lines and links are network segments. A new link is formed by the junction of no more than two links and sources are the points further upstream in the channel network. The magnitude of a link is the number of sources upstream draining into that link. The magnitude $n$ of the channel network is that of the outlet link, and equals the number of first-order streams. Topological distance $d$ (Figure 1 ) of a node from the outlet, or topological level of the link which originates from it to the downstream, is the number of links forming the path between that node and the outlet. The maximum of the topological distances within the network is called the topological diameter $D$. In Figure 1 the Horton-Strahler ordering scheme is also shown.

We consider a drainage basin as a system, that is of magnitude $n$; in other words, it has $M=2 n-1$ links. Let elevation $E$ be the state variable of the system. Each link is then associated with an average elevation and can have one of the $m$ average elevations $E_{i}, i=1,2, \cdots, m, m \leq M$. Following this representation of the drainage network, we first define the informational entropy of the network.

Let $p_{i}$ be the probability of a link having the elevation $E_{i}$. The informational entropy $S$ [Shannon, 1948] of the drainage basin system can be expressed as

$$
S=-\sum_{i=1}^{m} p_{i} \ln p_{i}
$$

By maximizing $S$ and applying the principle of maximum entropy [Jaynes, 1957], we can determine the probability distribution $P=\left(p_{1}, p_{2}, \cdots, p_{m}\right)$ subject to the given information on link elevations.

Let us suppose that the only information available on the drainage basin is the mean elevation $\bar{E}$ of all the links given by

$$
\vec{E}=\sum_{i=1}^{m} p_{i} E_{i}
$$

and, of course, on $p_{i}$ values as

$$
\sum_{i=1}^{m} p_{i}=1
$$

We assume that $\bar{E}$ is approximately equal to the mean basin elevation. There may be an infinity of probability distributions satisfying (2) and (3), for we have only two equations and $M(>2)$ quantities to be determined. Thus, following Jaynes [1957] the least biased $P$ is the one that maximizes $S$ in (1) subject to (2) and (3). Accordingly, invocation of the principle of maximum entropy yields

$$
p_{i}=\frac{\exp \left(-\lambda E_{i}\right)}{\sum_{i=1}^{m} \exp \left(-\lambda E_{i}\right)}
$$

where $\lambda$ is a Lagrange multiplier which can be determined from 


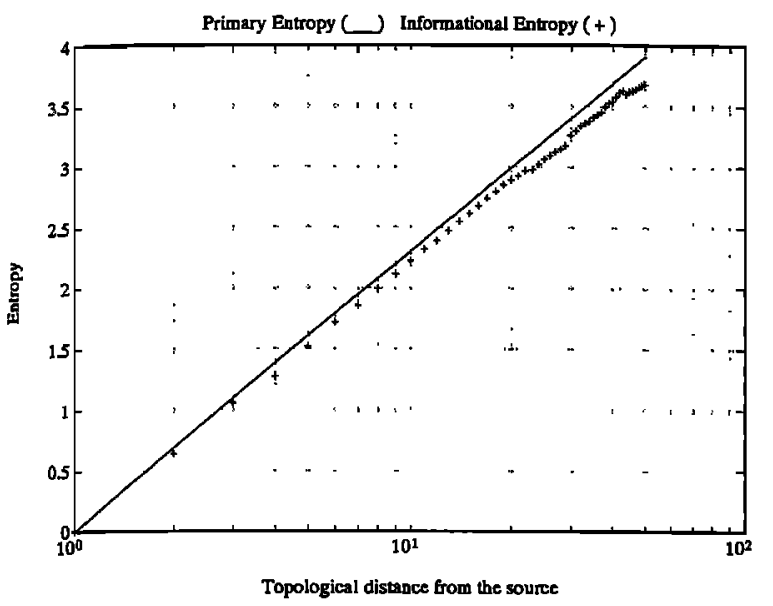

Fig. 2. Deviation of computed informational entropy (equation (1)) from primary entropy (equation (7)), for the main channel of Arcidiaconata basin.

$$
\frac{\sum_{i=1}^{m} E_{i} \exp \left(-\lambda E_{i}\right)}{\sum_{i=1}^{m} \exp \left(-\lambda E_{i}\right)}=\bar{E}
$$

The probability distribution given by (4) and (5) is incidentally the Maxwell-Boltzmann distribution. The maximum entropy $S_{\max }$ is given by substitution of (4) in (1):

$$
S_{\max }=\lambda \bar{E}+\ln \sum_{i=1}^{m} \exp \left(-\lambda E_{i}\right)
$$

Yet nothing has been stated about the way of choosing $E_{i}$ values. One way of accomplishing it that has hydrological appeal is to choose $E_{i}$ values as the mean elevations of nodes having the same topological distance from the basin outlet. Following this approach, $p_{i}$ values are the proportions of links at the $i$ th topological level, which also define the topological width function [Troutman and Karlinger, 1984] of the network. Under this scheme, all the previously derived equations hold with the topological diameter $D$ being equal to the possible number of states $m$.

We may now define the primary entropy of a drainage network as the value of the informational entropy when no constraints other than (3) are imposed. Then maximization of $S$ in (1) subject to (3) leads to

$$
S=\ln D, \quad p_{i}=\frac{1}{D}
$$

The entropy in (7) can be referred to as the primary entropy of the drainage basin. When constraints are imposed on the drainage basin such as mean elevation, and so on, its maximum entropy will be reduced. This reduced entropy can be called the secondary entropy which, for example, is given by (6). The difference between the primary and secondary entropies quantifies the reduction in uncertainty due to the knowledge of information expressed as constraints.

The maximum unconstrained value of entropy (primary) corresponds to a network with uniform topological width function. Although far from reality, the entropy of this simplified basin configuration provides a good approximation to the value of informational entropy produced by (6), as can be noted by Figure 2 that shows primary (equation (7)) and computed informational (equation (1)) entropies for subnetworks of the main channel of Arcidiaconata basin, a basin whose characteristics will be reported in the next section.

\section{Drainage Basin Data}

The data of three drainage basins, designated as Arcidiaconata, Lapilloso, and Vulgano, located in southern Italy, approximately at the forty-first parallel, were employed to verify the morphological analyses to be made in the subsequent sections. These basins are adjacent to each other and have the same climate. The region including these basins is semiarid. The average annual temperature of this region is about $14^{\circ} \mathrm{C}$, the average rainfall is about $720 \mathrm{~mm}$, and the average runoff coefficient is about 0.22 . Table 1 shows some pertinent characteristics of the three basins.

With the use of topographic maps (scale 1:25000), all the stream links existing within these basins were identified by use of blue lines. Each link would have its own drainage area and length. By using the contour lines, elevations of all the network nodes were computed. In elevation computations, each node was associated with the link which originated from it to the downstream. The links were ordered by topological level, and an array of connections was built. This scheme allowed identification of the subnetwork of a link and computation of the average elevation of the subnetwork with respect to its outlet elevation. Computations of entropy and network characteristics were made for all the subnetworks that numbered 516 for the three basins.

\section{Entropy and Potential Energy}

In stream morphology, potential energy plays a very important role. This energy is given by the elevation of the network nodes above a datum (say, the basin outlet), with proper consideration of scale units. For establishing a relation between entropy and potential energy, we consider $y_{d}$ as the mean node elevation at the topological distance $d$ from

TABLE 1a. Some Characteristics of Three Drainage Basins in Southern Italy

\begin{tabular}{lccccc}
\hline \multicolumn{1}{c}{ Basin } & $\begin{array}{c}\text { Surface Area, } \\
\mathrm{km}^{2}\end{array}$ & $\begin{array}{c}\text { Perimeter, } \\
\mathrm{km}\end{array}$ & $\begin{array}{c}\text { Drainage } \\
\text { Density, } \\
\mathbf{k m}^{-1}\end{array}$ & $\begin{array}{c}\text { First-Order } \\
\text { Stream } \\
\text { Frequency }\end{array}$ & $\begin{array}{c}\text { Mean Annual } \\
\text { Rainfall, mm }\end{array}$ \\
\hline Arcidiaconata & 123.9 & 59.5 & 2.24 & 2.05 & 770 \\
Lapilloso & 28.5 & 27.5 & 2.34 & 2.52 & 648 \\
Vulgano & 94.1 & 50.6 & 2.08 & 2.05 & 697 \\
\hline
\end{tabular}


TABLE $1 b$. Some Characteristics of Three Drainage Basins in Southern Italy

\begin{tabular}{lccccc}
\hline & $\begin{array}{c}\text { d, Mean } \\
\text { Topological } \\
\text { Level }\end{array}$ & $\begin{array}{c}n, \\
\text { Magnitude }\end{array}$ & $\begin{array}{c}R_{B}, \\
\text { Bifurcation } \\
\text { Ratio }\end{array}$ & $\begin{array}{c}\boldsymbol{R}_{L}, \text { Horton } \\
\text { Length Ratio }\end{array}$ & $\begin{array}{c}\text { Mean Annual } \\
\text { Runoff, mm }\end{array}$ \\
\hline Arcidiaconata & 25.1 & 254 & 4.12 & 2.39 & 188 \\
Lapilloso & 19.5 & 72 & 4.34 & 2.28 & 125 \\
Vulgano & 18.2 & 193 & 3.79 & 2.26 & 120 \\
\hline
\end{tabular}

the outlet. The value of $y_{d}$ is obtained by averaging the elevations of the nodes at distance $d$ from the outlet. We take $\bar{y}$ as the mean elevation for all the nodes and take it as approximately the mean basin elevation. The value $\bar{y}$ is the total potential energy of the drainage network system. The topological distance $d$ can vary from 1 to $D$, where $D$ is the network diameter. The current state of a drainage network can be surmised as the result of its history dating back to infinity. It may then be useful to evaluate the entropy of the network in its most probable state under the constraint imposed by the mean basin elevation. To that end, we write

$$
\begin{gathered}
\sum_{d=1}^{D} p_{d} y_{d}=\bar{y} \\
\sum_{d=1}^{D} p_{d}=1
\end{gathered}
$$

where $p_{d}$ is the probability of $M_{d}$ number of nodes having the mean elevation $y_{d}$. The probability distribution $P=\left\{p_{d}\right.$, $d=1,2, \cdots, D\}$ is given by (4), with $y_{d}$ replacing $E_{i}$ and $1 /(D T)$ replacing $\lambda$, as

$$
p_{d}=\frac{\exp \left[-\frac{y_{d}}{(D T)}\right]}{\sum_{d=1}^{D} \exp \left[-\frac{y_{d}}{(D T)}\right]}
$$

where $T$ is a parameter whose definition will become clear in the ensuing discussion. By analogy with a thermodynamic system, $T$ can be thought of as the (degenerate) temperature of the drainage network.

By reparameterizing (10), we obtain

$$
y_{d}=-\alpha \ln \left(\beta p_{d}\right)
$$

where

$$
\begin{gathered}
\alpha=D T \\
\beta=\sum_{d=1}^{D} \exp \left(-\frac{y_{d}}{\alpha}\right)
\end{gathered}
$$

By substituting (11) in (8) we obtain

$$
\bar{y}=-\alpha \sum_{d=1}^{D} p_{d} \ln \left(\beta p_{d}\right)
$$

or, using (1),

$$
\bar{y}=-\alpha \ln \beta+\alpha S
$$

This gives the relation for a drainage basin between its potential energy and entropy under the constraint in (8). This relation should also hold for any drainage subnetwork within the larger basin.

To verify (15), the average elevation and the entropy of the subnetwork of each link which is a part of the main stream were computed for the three drainage basins. The values of entropy were obtained by using (1). The average elevation of the subnetwork was plotted against its entropy, as shown in Figure 3 for the basins considered. Also plotted in the figure are the least squares lines obtained by treating $-\alpha \ln \beta$ and $\alpha$ as regression constants. For all the basins, (15) explained more than $80 \%$ of the variability in the average elevation, as shown in Table 2, where the regression constants and the coefficient of determination $R^{2}$ are reported.

For the drainage network system the distribution of potential energy can be assumed to be controlled by two fundamental quantities $D$ and $T$ measured for the entire system. On the basis of the analogy with a thermodynamic system where $T$ can be thought of as proportional to the energy content of the system, $\alpha$ and $\beta$ may be assumed to be constant as a first-order approximation. Thus $\alpha$ and $\beta$ can be theoretically derived as follows.

TABLE 1c. Some Characteristics of Three Drainage Basins in Southem Italy

\begin{tabular}{lcccc}
\hline & $\begin{array}{c}\bar{y} \text {, Nodal Mean } \\
\text { Elevation, m } \\
\text { amsl }\end{array}$ & $\begin{array}{c}\text { Nodal Maximum } \\
\text { Elevation, m } \\
\text { amsl }\end{array}$ & $\begin{array}{c}\text { Minimum Elevation, } \\
\text { m amsl }\end{array}$ & $\begin{array}{c}H, \text { Total Fall } \\
\text { in Elevation, m }\end{array}$ \\
\hline Arcidiaconata & 538 & 894 & 237 & 657 \\
Lapilloso & 547 & 819 & 319 & 500 \\
Vulgano & 568 & 862 & 199 & 663 \\
\hline
\end{tabular}

Here amsl is above mean sea level. 

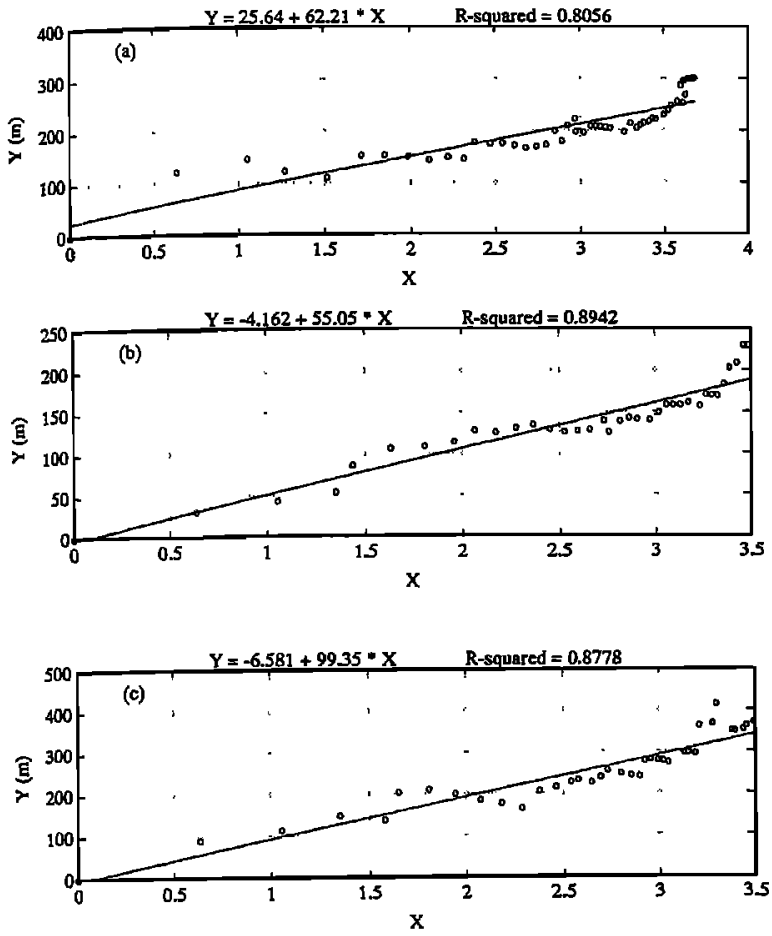

Fig. 3. Average elevation $\bar{y}_{\delta}(Y)$ versus informational entropy $S_{\delta}(X)$ of subnetworks whose outlets lie on the main channel and respective least squares lines, for (a) Arcidiaconata, (b) Lapilloso, and $(c)$ Vulgano basins.

The primary entropy for a drainage network of diameter $D$ is given by the first of (7),

$$
S=\ln D
$$

Similarly, for a drainage subnetwork of diameter $\delta$ the primary entropy is

$$
S_{\delta}=\ln \delta
$$

Let $\bar{y}_{\delta}$ be the mean elevation of the drainage subnetwork of diameter $\delta$ whose outlet lies on the channel of diameter $D$. Let $\bar{y}$ be the mean elevation of the entire network of diameter $D$. At the basin source, $\bar{y}_{\delta}=0$ and $S=0$. In (15), then, $\ln \beta=0$, which yields $\beta=1$; equation (15) therefore becomes

$$
\bar{y}=\alpha S
$$

Application of (16) to (18) yields

$$
\alpha=\frac{\bar{y}}{S}=\frac{\bar{y}}{\ln D}
$$

Since (18) is valid for any drainage subnetwork of diameter $\delta$, it can be written as

TABLE 2. Least Squares Estimates of Coefficients in Equation (15)

\begin{tabular}{lcccc}
\hline \multicolumn{1}{c}{ Basin } & $-\alpha \ln \beta$ & $\alpha$ & $\beta$ & $R^{2}$ \\
\hline Arcidiaconata & 25.64 & 62.21 & 0.66 & 0.805 \\
Lapilloso & -4.162 & 55.05 & 1.08 & 0.894 \\
Vulgano & -6.581 & 99.35 & 1.07 & 0.878 \\
\hline
\end{tabular}
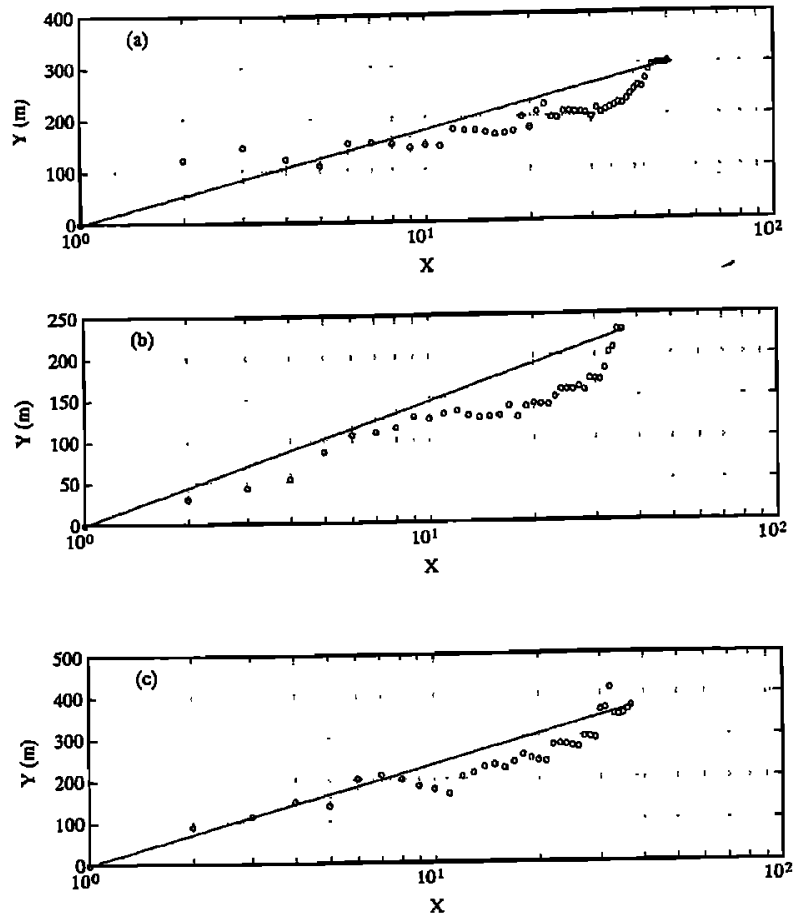

Fig. 4. Average elevation $\bar{y}_{\delta}(Y)$ versus topological diameter $\delta(X)$ of subnetworks whose outlets lie on the main channel, compared with the lines representing equation (21), for (a) Arcidiaconata, (b) Lapilloso, and (c) Vulgano basins.

$$
\bar{y}_{\delta}=\alpha S_{\delta}
$$

which, with substitution of $\alpha$ obtained from (19), becomes

$$
\bar{y}_{\delta}=\bar{y} \frac{\ln \delta}{\ln D}
$$

This establishes the relation between the mean elevation of a drainage subnetwork and its diameter $\delta$. With use of (12) and (19), parameter $T$ is found to be

$$
T=\frac{\bar{y}}{D \ln D}
$$

This defines what was referred to as the (degenerate) temperature of the drainage network. It is proportional to the potential energy of the system and inversely proportional to a coefficient that monotonically increases with the diameter of the drainage network. Equation (22) provides a quantitative measure of the macroscopic thermodynamical property of the system.

The data of the three basins were used to evaluate the adequacy of (21). Figure 4 shows the relation between the average elevation of a subnetwork and its topological diameter for the three basins. For each basin the amount of variance explained in the average elevation of the subnetwork was greater than $75 \%$. It may be noted that part of the unexplained variance may be ascribed to the use of topological distances instead of actual distances. The observation that link lengths tend to increase in the downstream direction suggests that the graphs of Figure 4 would spread in the $x$ direction if the real distances were considered. Further investigations are needed to quantify this spread. To sum- 


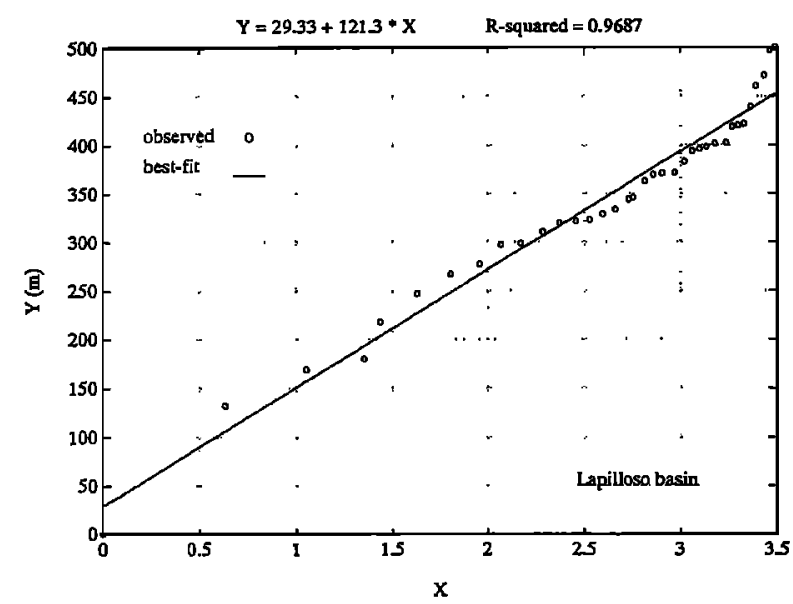

Fig. 5. Elevation drop $H_{\delta}$ from the source $(Y)$ versus entropy $S_{\delta}(X)$ for the main channel of Lapilloso basin.

marize, with use of the basin planimetric configuration, (21) can be reliably used for estimation of subnetwork mean elevation when elevations are used for investigating such network characteristics as channel profiles and scaling properties of slopes. This matter will be treated further in the next sections.

\section{Longitudinal Channel Profile}

The longitudinal bed profile of a channel can be derived by employing (15) and (16) and by considering (8). In the spirit of using primary entropy as an approximation of the thermodynamical entropy, a uniform width function provides a first-order approximation for $p_{i}$ values as being equal to $1 / D$. Furthermore, invoking the principle of equal energy expenditure, which is analogous to the concepts used by Yang [1971] and Rodriguez-Iturbe et al. [1992], the network elevation drop may be assumed to be uniformly distributed between topological levels. That means mean elevation drops between two consecutive levels are the same. Therefore, following (8), the total elevation fall $H_{\delta}$ from the source to the outlet of a channel of diameter $\delta$ is given by

$$
H_{\delta}=\frac{\delta \bar{y}_{\delta}}{\bar{d}_{\delta}}
$$

where $\bar{y}_{\delta}$, as before, denotes the mean elevation of the subnetwork, and $\bar{d}_{\delta}$ is the topological distance of the centroid of the sub-basin width function.

By multiplying both sides of (15) by $\delta / \bar{d}_{\delta}$ we get

$$
H=-\alpha^{\prime} \ln \beta+\alpha^{\prime} S
$$

where $\alpha^{\prime}=\alpha \delta / \bar{d}_{\delta}, \alpha$ is defined by (12), and $H$ is the fall in elevation from the source to the outlet of the main channel of the drainage network whose entropy is $S$. The values of $H$ and $S$, obtained for the three basins considered, were used to verify (24). By treating $-\alpha^{\prime} \ln \beta$ and $\alpha^{\prime}$ as regression constants, the best fit lines were obtained for the plots of the elevation drop against entropy for each link of the main stream, as shown in Figure 5 for Lapilloso basin. For all the basins, (24) explained more than $90 \%$ of the variance in the fall in elevation, as shown in Table 3.
TABLE 3. Least Squares Estimates of Coefficients in Equation (24)

\begin{tabular}{lccc}
\hline \multicolumn{1}{c}{ Basin } & $-\alpha^{\prime} \ln \beta$ & $\alpha^{\prime}$ & $R^{2}$ \\
\hline Arcidiaconata & -2.057 & 167 & 0.966 \\
Lapilloso & 29.33 & 121 & 0.968 \\
Vulgano & -104.3 & 184 & 0.910 \\
\hline
\end{tabular}

Parameters $\alpha^{\prime}$ and $\beta$ can be theoretically evaluated as was done for (15). Accordingly, (24) provides

$$
H_{\delta}=a^{\prime} S_{\delta}=\frac{H}{\ln D} S_{\delta}
$$

Let $y_{0}$ be the elevation of the source of the channel and $y_{\delta}$ be the elevation of the downstream node at a distance $\delta$ from the source. Then, with substitution of (17) in (25), the bed elevation profile of a channel of topological length $D$ is given by

$$
y_{\delta}=y_{0}-H \frac{\ln \delta}{\ln D}
$$

This gives the relation between elevation at a point and its topological distance. This relation was plotted for the drainage basins under consideration. For each basin, more than $95 \%$ of the variability in the nodal elevation was explained by (26). Figure 6 shows the relation for Lapilloso basin.

\section{Entropy, Horton-Strahler Order, Magnitude, AND FRactal DIMENSION}

We consider a drainage network of Horton-Strahler order $\boldsymbol{\Omega}$. In the Horton-Strahler ordering scheme a channel of the order of $\omega, 2 \leq \omega \leq \Omega$, is defined as the succession of links of the order of $\omega$ whose last link drains either into a link of the order of $\omega^{*}>\omega$ or into the outlet. The drainage basin has the bifurcation ratio $R_{B}$ and stream-length ratio $R_{L}$ defined as

$$
R_{B}=\frac{N_{\omega-1}}{N_{\omega}}
$$

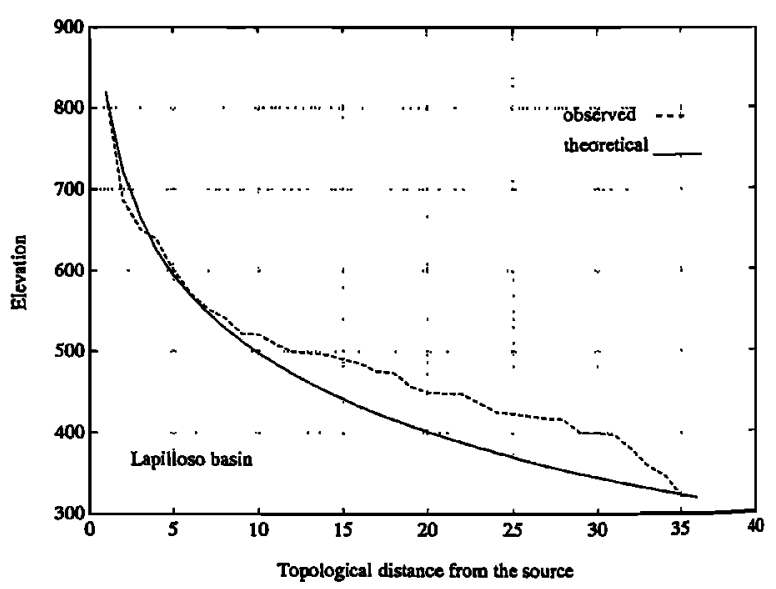

Fig. 6. Relation between nodal elevation and topological distance from the source for the main channel of Lapilloso basin. The theoretical line refers to equation (26). 
TABLE 4. Relation Between Entropy and Logarithm of Magnitude

\begin{tabular}{lccllcrr}
\hline & \multicolumn{2}{c}{ Equation (34) Coefficients } & & \multicolumn{2}{c}{ Least Squares Coefficients } \\
\cline { 2 - 3 } \multicolumn{1}{c}{ Basin } & $\ln R_{L} / \ln T_{B}$ & $\ln \left[R_{L} /\left(R_{L}-1\right)\right]$ & & Slope & Intercept & $R^{2}$ \\
\hline Arcidiaconata & 0.616 & 0.541 & & 0.614 & 0.417 & 0.952 \\
Lapilloso & 0.575 & 0.562 & & 0.770 & 0.088 & 0.978 \\
Vulgano & 0.633 & 0.562 & & 0.539 & 0.645 & 0.964 \\
\hline
\end{tabular}

Least squares estimates and computed theoretical coefficients of equation (34)

$$
R_{L}=\frac{L_{\omega}}{L_{\omega-1}}
$$

where $N_{\omega}$ is the number of streams of the order of $\omega$ in the drainage basin network of order $\Omega$, and $L_{\omega}$ is the average length of streams of the order of $\omega$.

In a Hortonian network the topological diameter $D$ can be expressed in terms of the stream-length ratio as

$$
D=\sum_{\omega=1}^{\Omega} R_{L}^{\omega-1}=\frac{1-R_{L}^{\Omega}}{1-R_{L}}=\frac{R_{L}^{\Omega}-1}{R_{L}-1}
$$

By taking the logarithm of (29) we get

$$
\ln D=\ln \left[\frac{R_{L}^{\Omega}-1}{R_{L}-1}\right]
$$

which, by virtue of (16), is the entropy of the HortonStrahler drainage network of the order of $\Omega$. Therefore

$$
S=\ln \left(R_{L}^{\Omega}-1\right)-\ln \left(R_{L}-1\right)
$$

A simplification of (31) can be achieved as follows. Note that $R_{L}>1$, and if $\Omega$ is much greater than 1 , then $R_{L}^{\Omega}-1 \approx$ $R_{L}^{h}$. Under this condition, (31) simplifies to

$$
S=\Omega \ln R_{L}-\ln \left(R_{L}-1\right)
$$

This establishes a linear relation between entropy and the drainage basin order. Thus the Horton-Strahler order can be thought of as a measure of network complexity. This reinforces the findings of Wang and Waymire [1991].

The magnitude $n$ of a drainage basin network is one of its most important characteristics, for it provides a surrogate for the drainage area. In terms of the bifurcation ratio the magnitude can be expressed as

$$
\begin{gathered}
n=R_{B}^{\Omega-1} \\
\Omega=1+\frac{\ln n}{\ln R_{B}}
\end{gathered}
$$

By applying (33) to eliminate $\Omega,(32)$ can be expressed as

or

$$
S=\frac{\ln R_{L}}{\ln R_{B}} \ln n+\ln \left[\frac{R_{L}}{R_{L}-1}\right]
$$

$$
S=(\Omega-1) \ln R_{L}+\ln \left[\frac{R_{L}}{R_{L}-1}\right]
$$

Equations (34) and (35) express the relation between entropy $S$, magnitude $n$, and the Horton order $\Omega$, respectively, of a drainage basin.

When the average entropy for fixed magnitude $n$ was plotted against the logarithm of $n$, a strong linear relation was found for all the basins, in agreement with (34), with coefficients of determination $R^{2}$ always greater than 0.95 . Both the angular coefficient and the intercept of the least squares lines were close to the theoretical coefficients of (34) (see Table 4). Figure 7 shows the entropy-magnitude relationship for the Arcidiaconata basin.

The average entropy for fixed Horton order $\omega$ was plotted against $\omega$ and a strong linear relation was also found, with $R^{2}$ greater than 0.99 for all the basins (Table 5). However, the intercept value significantly deviated from the value of the parameter $\ln \left[R_{L} /\left(R_{L}-1\right)\right]$ appearing in (35). Figure 8 shows the entropy-Horton order relationship for the Arcidiaconata basin. It may be concluded that (34) and (35) seem well suited to explain the variability of the entropy with the magnitude and Horton order respectively, even though more investigations are needed to identify the reasons of the inaccuracy of the second term at the right-hand side of (35).

To further explore the entropic properties of drainage networks, the cumulative entropy was computed as the sum of informational entropies of Horton streams having the same order for each of the three basins under consideration, and it was plotted against the Horton order. The plot is shown in Figure 9 for Arcidiaconata basin. Clearly, there is a strong relation between the logarithm of cumulative en-

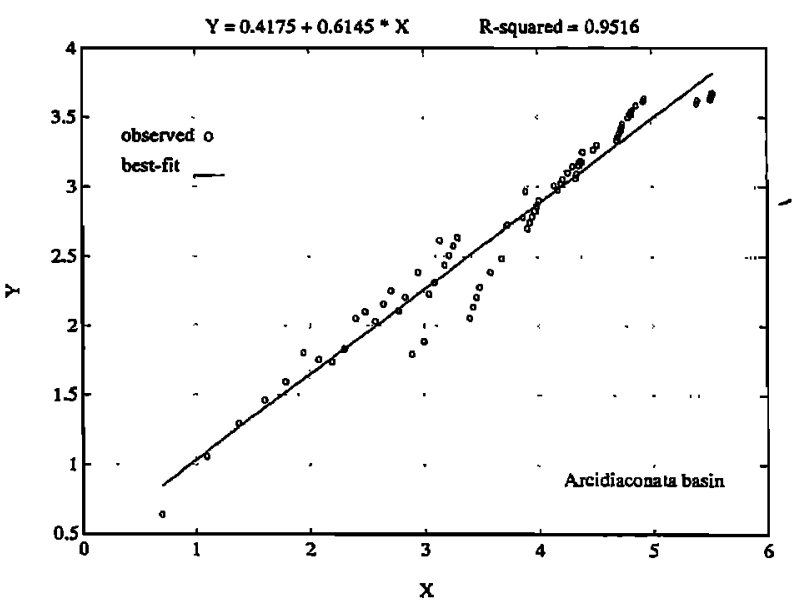

Fig. 7. Relation between the average entropy $(Y)$ and logarithm of magnitude $(X)$ for Arcidiaconata basin. 
TABLE 5. Relation Between Entropy and the Horton Order

\begin{tabular}{lcccccc}
\hline & \multicolumn{2}{c}{ Equation (35) Coefficients } & & \multicolumn{3}{c}{ Least Squares Coefficients } \\
\cline { 2 - 3 } \multicolumn{1}{c}{ Basin } & $\ln R_{L}$ & $\ln \left[R_{L} /\left(R_{L}-1\right)\right]$ & & Slope & Intercept & $R^{2}$ \\
\hline Arcidiaconata & 0.872 & 0.541 & & 0.943 & 0.08 & 0.990 \\
Lapilloso & 0.845 & 0.562 & & 1.143 & 0.08 & 0.998 \\
Vulgano & 0.844 & 0.562 & & 0.852 & -0.14 & 0.990 \\
\hline
\end{tabular}

Least squares estimates and computed theoretical coefficients of equation (35).

tropy and the Horton order. The value of the intercept at zero of the best fit line representing this relation was interestingly close to the total number of links in the basin. Figure 9 suggests that the relation between cumulative entropy and the Horton order can be expressed meaningfully as

$$
S_{\Sigma}=(2 n-1) \exp (-\omega)=M \exp (-\omega)
$$

where $S_{\Sigma}$ is the cumulative entropy. This shows that when $\omega$ $=0, S_{\Sigma}=(2 n-1)=M$ is the total number of links in the basin. In Table 6 the actual values of $M$ are compared with those estimated by (36) using $M$ as a regression coefficient. For the two larger basins (fifth order) the differences between actual and estimated values of $M$ are significantly small.

\section{Scaling Properties}

A well-known empirical relation between the length $L$ of the main channel and the drainage area $A$ is

$$
L \propto A^{\oplus}
$$

in which $\Theta$ was found to be, on average, 0.568 by Gray [1961] and 0.554 by Mueller [1973]. Mandelbrot [1983] used (37) to derive the fractal dimension $\left(D_{l}\right)$ of channels, $D_{l}=$ $2 \Theta \approx 1.14$. La Barbera and Rosso [1989] defined the fractal dimension of stream networks as

$$
F=\frac{\ln R_{B}}{\ln R_{L}}
$$

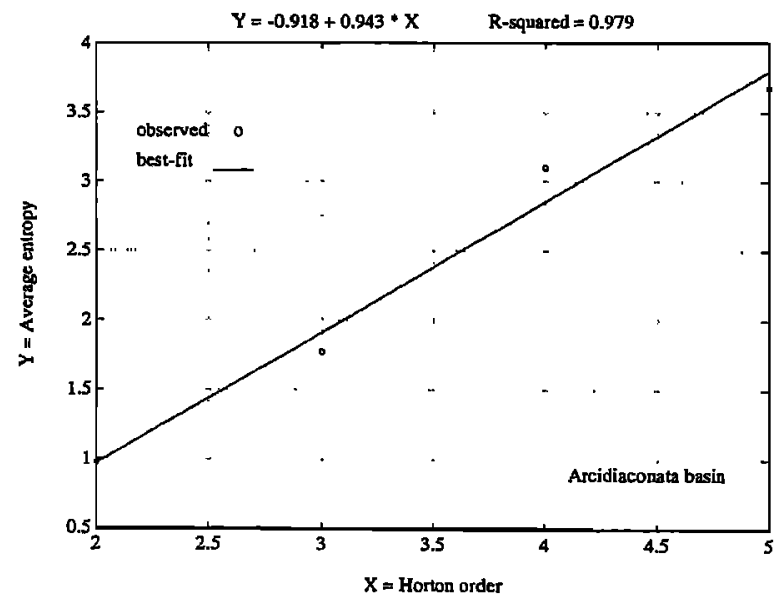

Fig. 8. Relation between the average entropy and the Horton order for Arcidiaconata basin.
Tarboton et al. [1990] recognized $F$ to be the fractal dimension due to the branching process, with the product $F D$ being the total fractal dimension of the stream network systems. Thus, according to Mandelbrot's value of $D_{l}, F$ should be equal to 1.75 for space-filling river systems. A relation analogous to (37) can be derived by equating (16) to (34) as

$$
D=C n^{1 / F}
$$

where $C=R_{L} /\left(R_{L}-1\right)$. Equation (39) is in an obvious analogy with (37) and sheds more light on the meaning of $\Theta$, which comes out as being equal to $1 / F$. For $F=1.75$, (39) leads to $\Theta=0.57$, which is in excellent agreement with Gray [1961].

Substitution of (39) in (34) produces

$$
S=\ln \left(\frac{R_{L}}{R_{L}-1} n^{1 / F}\right)
$$

which represents the link between entropy, magnitude, and fractal dimension of networks.

Values of $S$ were plotted against the values of logarithms of magnitude $\boldsymbol{n}$ for various values of the stream-length ratio and bifurcation ratio, as shown in Figure 8. Also shown in the figure are the values of $S$ computed for Lapilloso basin. $S$ was obtained by averaging of the values of all the subbasins of the same magnitude. Given the variance in $R_{L}$ and $R_{B}$ found for a drainage basin, the agreement between the observed and the computed values of $S$ against $n$ is quite good.

Another important relation that has been a subject of a

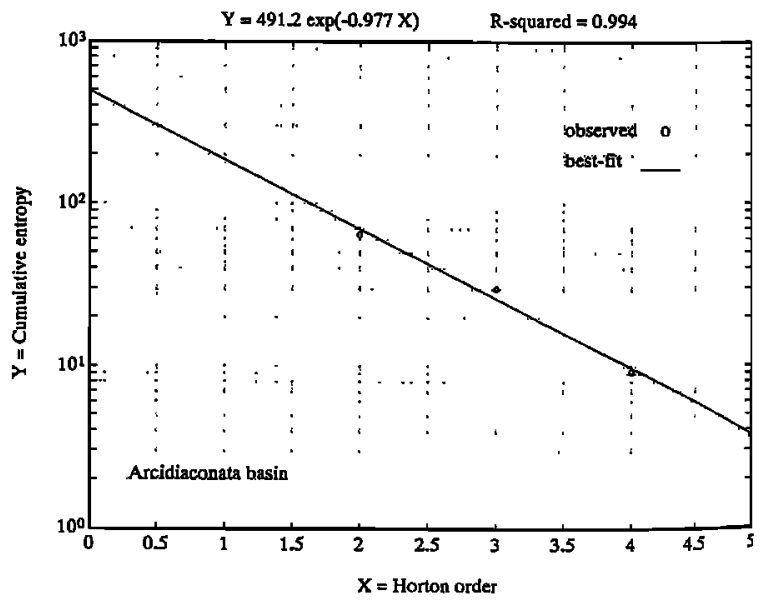

Fig. 9. Relation between cumulative entropy and the Horton order for Arcidiaconata basin. 
TABLE 6. Actual $(=2 n-1)$ and Estimated (Equation (36)) Total Number $M$ of Links

\begin{tabular}{lcc}
\hline Basin & Actual & Estimated \\
\hline Arcidiaconata & 507 & 488 \\
Lapilloso & 143 & 77 \\
Vulgano & 385 & 311 \\
\hline
\end{tabular}

number of investigations [e.g., Gupta and Waymire, 1989; Willgoose et al., 1991], is the one between channel slope $s$ and drainage area $A$,

$$
s \propto A^{-\gamma}
$$

with $\gamma$ varying from 0.37 to 0.83 , with an average of 0.60 [Flint, 1974]. The entropy-based river profile derived in section 5 provides an explanation for (41). To that end, by differentiating (26), we get

$$
s \propto \frac{1}{D}
$$

and, by virtue of (39),

$$
s \propto n^{-1 / F}
$$

which, for the analogy between $n$ and $A$, leads to $\gamma=1 / F$. For $F=1.75, \gamma=0.57$, which is very close to the average value observed by Flint [1974].

\section{CONClusions}

The following conclusions are drawn from this study:

1. A definition of the informational entropy $S$ of river networks was proposed. $S$ was shown to be capable of describing some planimetric and altimetric characteristics of network structures.

2. The primary entropy, which is the maximum unconstrained value of $S$, is given by the logarithm of topological diameter of the network. It provided a good first approximation for the informational entropy and allowed us to establish, using entropy concepts, relations between the main channel length and other network characteristics.

3. The potential energy and entropy are linearly related. This relation led to a linear relation between the mean elevation of a drainage subnetwork and the logarithm of its diameter.

4. The fall in elevation of the main channel and the entropy of its drainage basin are linearly related. This relation led to a river profile equation.

5. For a Horton-Strahler drainage network the entropy of the network was found to be linearly related to the network order

6. The entropy of a drainage network was found to be linearly related to the logarithm of magnitude $n$, with an angular coefficient given by the reciprocal of the fractal dimension $F$ of the network branching.

7. Two widely known empirical scaling properties of channels with surface area $A$ of the basin were theoretically derived. In particular, the length $L$ and slope $s$ of the main channel were shown to be proportional to $A^{\Theta}$ and to $A^{-\gamma}$ respectively, with $\Theta=\gamma=1 / F$.
Acknowledgments. This work was supported by funds granted by the Italian Ministero della Ricerca Scientifica e Tecnologica, Progetto 60\% "Criteri di massima entropia in idrologia e idraulica" and Progetto 40\% "Fenomeni di trasporto nel ciclo idrologico." Comments and suggestions by two anonymous referees are also acknowledged and faithfully appreciated.

\section{REFERENCES}

Boltzmann, L., Weitere Studien uber das warmegleich-gewich unter gasmolekulen, K. Acad. Wiss. (Wein) Sitzb., II Abt, p. 66, 1872.

Flint, J. J., Stream gradient as a function of order, magnitude and discharge, Water Resour. Res., 10(5), 969-973, 1974.

Gray, D. M., Interrelationships of watersheds characteristics, $J$. Geophys. Res., 66, 1215-1223, 1961.

Gupta, V. K., and E. Waymire, Statistical self-similarity in river networks parametrized by elevation, Water Resour. Res., 25(3), 463-476, 1989.

Gupta, V. K., C. T. Wang, and E. Waymire, A representation of an instantaneous unit hydrograph from geomorphology, Water Resour. Res., I6(5), 855-862, 1980.

Gupta et al. (Eds.), Scale Problems in Hydrology, D. Reidel, Hingham, Mass., 1986.

Jaynes, E. T., Information theory and statistical mechanics, I, Phys. Rev., 106, 620-630, 1957.

Kapoor, V., Spatial uniformity of power and the altitudinal geometry of river networks, Water Resour. Res., 26(1), 2303-2310, 1990.

La Barbera, P., and R. Rosso, On the fractal dimension of stream networks, Water Resour. Res., 25(4), 735-741, 1989.

Leopold, L. B., and W. B. Langbein, The concept of entropy in landscape evolution, U.S. Geol. Surv. Prof. Pap., 500-A, A-20 pp., 1962.

Lienhard, J. H., A statistical mechanical prediction of the dimensionless unit hydrograph, J. Geophys. Res., 69, 52-31, 1964.

Mandelbrot, B. B., The Fractal Geometry of Nature, W. H. Freeman, New York, 1983.

Marani, A., R. Rigon, and A. Rinaldo, A note on fractal channel networks, Water Resour. Res., 27(12), 3041-3049, 1991.

Mueller, J. E., Re-evaluation of the relationship of master streams and drainage basins: Reply, Geol. Soc. Am. Bull., 84, 3127-3130, 1973.

Prigogine, I., Introduction to Thermodynamics of Irreversible Processes, 3rd ed., John Wiley, New York, 1967.

Rodriguez-Iturbe, I., and J. B. Valdes, The geomorphologic structure of the hydrologic response, Water Resour. Res., 15(6), 1409-1420, 1979.

Rodriguez-Iturbe, I., A. Rinaldo, R. Rigon, R. L. Bras, A. Marani, and E. Ijjasz-Vasquez, Energy dissipation, runoff production and the 3-dimensional structure of river basins, Water Resour. Res., 28(4), 1095-1 103, 1992.

Rosso, R., B. Bacchi, and P. La Barbera, Fractal relation of mainstream length to catchment area in river networks, Water Resour. Res., 27(3), 381-387, 1991.

Scheidegger, A. E., Some implications of statistical mechanics in geomorphology, Int. Assoc. Sci. Hydrol. Bull., 9(1), 12-16, 1964.

Shannon, C. E., The mathematical theory of communications, I and II, Bell Sys. Tech. J., 27, 379-423, 1948.

Singh, V. P., Hydrologic modeling using entropy, J. Inst. Eng. Civ. Eng. Div., 70, Part CV2, 55-60, 1989.

Singh, V. P., and M. Fiorentino, A historical perspective of entropy applications in water resources, in Entropy and Energy Dissipation in Water Resources, edited by Singh and Fiorentino, pp. 21-61, Kluwer, Boston, Mass., 1992.

Song, C. C. S., and C. T. Yang, Variational method in fluvial hydraulics-A review, in Hydraulic Engineering, edited by $\mathrm{H} . \mathrm{H}$. Chang and J. C. Hill, pp. 299-304, American Society of Civil Engineers, New York, 1990.

Sonuga, J. O., Principle of maximum entropy in hydrologic frequency analysis, J. Hydrol., I7(3), 177-219, 1972

Tarboton, D. G., R. L. Bras, and I. Rodriguez-Iturbe, The fractal nature river networks, Water Resour. Res., 24(8), 1317-1322, 1988.

Tarboton, D. G., R. L. Bras, and I. Rodriguez-Iturbe, Comment on "On the fractal dimension of stream networks," by P. La Barbera and R. Rosso, Water Resour. Res., 26(9), 2243-2244, 1990.

Troutman, B. M., and M. R. Karlinger, On the expected width 
function for topologically random channel networks, J. Appl. Probab., 21, 836-849, 1984.

Wang, S. X., and E. C. Waymire, A large deviation rate and central limit theorem for Horton ratios, SIAM J. Discrete Math., 4(4), 575-588, 1991.

Willgoose, G., R. L. Bras, and I. Rodriguez-Iturbe, A physical explanation of observed link area-slope relationship, Water $R e-$ sour. Res., 27(7), 1697-1702, 1991.

Wilson, A. G., The use of the concept of entropy in system modeling, Oper. Res. Q., 21(2), 247-265, 1970.

Yang, C. T., Potential energy and stream morphology, Water Resour. Res., 7(2), 311-322, 1971.
P. Claps and M. Fiorentino, Dipartimento di Ingegneria e Fisica dell'Ambiente, Università della Basilicata, Via della Tecnica 3-85100 Potenza, Italy.

V. P. Singh, Department of Civil Engineering, Louisiana State University, Baton Rouge, LA 70803.

(Received December 12, 1991

revised September 15, 1992;

accepted September 28, 1992.) 